\title{
Association between obesity, dyslipidemia and insulin resistance with suspected fatty liver disease in Greek children with excess weight
}

\begin{abstract}
Objective: This study investigates the association between dyslipidemia and fatty liver disease with body mass index (BMI), waist-to-height ratio (WHtR), waist circumference (WC) and Homeostasis Model Assessment of Insulin Resistance (HOMA-IR) in children and adolescents with overweight or obesity.
\end{abstract}

Methods: Data from 299 children with overweight or obesity (48\% boys) with mean age $9.23 \pm 2.5$ years were analyzed. Alanine aminotransferase (ALT) $>25.8 \mathrm{U} / \mathrm{L}$ (boys) and $>22.1$ $\mathrm{U} / \mathrm{L}$ (girls), was defined as abnormal. Student's t-tests were computed for the comparison of mean values. Chi-square tests were used for the comparison of proportions.

Results: Proportions of children with dyslipidemia and suspected nonalcoholic fatty liver disease (NAFLD) were $23.4 \%$ and $31.4 \%$ respectively. The percentage of children with NAFLD was $28.3 \%$ in those without dyslipidemia and $45 \%$ in those with dyslipidemia $(\mathrm{p}=0.015)$. BMI, WC and WHtR were not different between children with and without dyslipidemia. BMI was significantly higher in those with NAFLD $(\mathrm{p}=0.019)$. A stratified by gender analysis, showed that BMI, WC and WHtR were significantly higher in boys with NAFLD ( $p=0.009,0.037,0.049$ respectively). WHtR $\geq 0.5$ and HOMA-IR $\geq 3$ were not significantly associated with the presence of dyslipidemia ( $p>0.999,0.549$ respectively), however HOMA-IR $\geq 3$ was more frequent in children with NAFLD $(\mathrm{p}=0.011)$. A stratified by gender analysis, showed that the aforementioned association was evident only in boys $(\mathrm{p}=0.027)$

Conclusion: The severity of obesity and male gender are clinical indicators of increased risk of dyslipidemia and NAFLD among obese children and adolescents.

Keywords: obesity, BMI, non-alcoholic fatty liver disease (NAFLD), metabolic syndrome (MetS), dyslipidemia
Volume 10 Issue 6 - 2020

\section{E Dikaiakou,' E Vlachopapadopoulou,' A Fotiadou, ${ }^{2} \mathrm{~F}$ Athanasouli,' $\mathrm{M}$ Kafetzi, ${ }^{3} \mathrm{~A}$ Fotinou, ${ }^{3}$ S Michalacos' \\ 'Department of Endocrinology-Growth and Development, Children's Hospital P. \& A. Kyriakou, Greece 2Department of Paediatrics, University of Athens, Greece 'Biochemistry Department, HormonesLaboratory, Children's Hospital P. \& A. Kyriakou, Greece}

Correspondence: Elpis Athina Vlachopapadopoulou, Division of Endocrinology, Growth and Development Children's Hospital, "P.\&A. Kyriakou",Athens, Greece, Tel+30 2/3200985 I/+30 6932247228; Fax +30 2132009531, Email elpis.vl@gmail.com

Received: November 08, 2020 | Published: November 25, 2020
Abbreviations: ALT, alanine aminotransferase; BMI, body mass index; HDL, high-density lipoprotein; HOMA-IR, homeostasis model assessment of insulin resistance; IR, insulin resistance; LDL, low-density lipoprotein; NAFLD, nonalcoholic fatty liver disease; NASH, nonalcoholic steatohepatitis; OR, odds ratio; SPSS, statistical package for the social sciences; Triglycerides; TC, total cholesterol; WC, waist circumference; WHO, World health organization; WHtR, waist-to-height ratio

\section{Introduction}

Overweight and obesity is dramatically rising worldwide according to the World Health Organization (WHO). ${ }^{1}$ Pediatric obesity is strongly associated with substantial comorbidities including insulin resistance (IR), metabolic syndrome (MetS), long-term vascular complications and fatty liver. ${ }^{1}$ Nonalcoholic fatty liver disease (NAFLD) is defined as the accumulation of fat in the liver in the absence of excessive alcohol consumption or other known pathologies. The term refers to a wide spectrum of liver disease, ranging from asymptomatic steatosis to nonalcoholic steatohepatitis (NASH), that includes hepatic inflammation and can result in various degrees of fibrosis and cirrhosis, even in children. ${ }^{2}$ NAFLD is the most common chronic liver disease in children; it is a multifactorial disorder and the most common indication for liver transplantation in young adults. ${ }^{2}$ NAFLD is considered the hepatic manifestation of MetS and is associated with several components of MetS, such as central obesity, IR and dyslipidemia. ${ }^{3,4}$ There are several methods to diagnose NAFLD, such as imaging for steatosis (ultrasound, computed tomography, magnetic resonance imaging), liver biopsy and liver enzymes function. The exact diagnostic accuracy of ALT is not well established, however, ALT is the most commonly used screening tool for NAFLD. ${ }^{5}$ Liver biopsy remains the gold standard to diagnose NAFLD, but it is highly invasive in children and expensive. NASPGHAN guidelines suggest ALT as the recommended first line test for NAFLD in children as it mainly correlates with the presence of steatosis. ${ }^{6}$ ALT should be used as a screening tool and not as a diagnostic tool. ${ }^{6}$ This study explores the association between dyslipidemia and (NAFLD) with BMI, waist-toheight ratio (WHtR), waist circumference (WC) and insulin resistance index HOMA-IR in overweight and obese children and adolescents.

\section{Materials \& methods}

\section{Study design and population}

The study group included 299 children (48 \% boys), who were investigated, in the Department of Endocrinology, Growth and Development, "P. \& A. Kyriakou" Children's Hospital, Athens, Greece between 2013 and 2016. All the patients were referred for investigation and treatment of increased body weight. Their mean age was $9.23 \pm 2.5$ years. Children were excluded from the analysis, if they 
had obesity related syndromes, type 1 or type 2 diabetes mellitus, long-term corticosteroid use, primary hyperlipidemia, as well as any other reason for hepatic steatosis, such as medication (amiodarone, L-asparaginase, valproic acid), cystic fibrosis, HIV, hepatitis B or C, Wilson's disease or celiac disease.

Weight and height were measured to the nearest $0.1 \mathrm{~kg}$ and $0.1 \mathrm{~cm}$, respectively, with the subjects standing barefoot and in light clothing. Waist circumference (WC) was measured at the level of midway between the lowest rib and the top of the iliac crest. All measurements were taken twice, and the two measurements were averaged for analysis. BMI was calculated as weight (in kilograms) divided by height (in meters) squared. Childhood obesity was defined as BMI equal to or greater than the sex- and age-specific $95^{\text {th }}$ percentile of CDC Anthropometric Reference Data for Children and Adults, 2007$2010 .{ }^{1}$ Moreover, a child or adolescent $\geq 2$ years of age was considered as overweight if the BMI was $\geq 85$ th percentile but $<95$ th percentile. Weight to Height ratio (WHtR) was calculated by dividing WC by height. Abdominal obesity was defined as WHtR $\geq 0.5$ and $\mathrm{WC}$ equal to or greater than the sex- and age-specific $90^{\text {th }}$ percentile. ${ }^{7}$

\section{Assays}

After an overnight fast, glucose, lipid profile, Aspartate aminotransferase (AST), ALT and insulin levels were measured. HOMA-IR index was calculated in all participantsas fasting plasma insulin (FPI U/l) $\times$ fasting plasma glucose (FPG mg/dl)/405. A cut-off value $\geq 3$ was used for HOMA-IR. ${ }^{8,9}$ There is no single standard cut off point for abnormal serum aminotransferase in children's population. ALT $>25.8 \mathrm{U} / \mathrm{L}$ (boys) and $>22.1 \mathrm{U} / \mathrm{L}$ (girls) was defined as abnormal in this research, in accordance with SAFETY study. ${ }^{10}$

Venous samples were collected in BD Vacutainer ${ }^{\circledR}$ spray coated K2EDTA tubes and in WEGO serum vacuum tubes. Plasma glucose levels, AST and fasting serum lipids [serum triglycerides (Tg), total cholesterol, high-density lipoprotein (HDL) cholesterol, and lowdensity lipoprotein (LDL) cholesterol], were measured not more than 10 minutes after the blood drawn by an enzymatic, colorimetric method in a Cobas c501 autoanalyzer (Roche Laboratory Systems). The intra and inter-assay coefficient variability (CV) with this method of glucose measurement in our laboratory is less than 3.2\%. Fasting insulin was measured using the immunometric reaction, ECLIA (Electrochemiluminescence method) Cobas c601 Roche Diagnostics, Greece, all conducted in a CLIA (Clinical Laboratory Improvement Amendments) approved laboratory.

\section{Statistical analysis}

Statistical analyses were performed using SPSS software (version 22.0). Quantitative variables are presented with mean values and standard deviation (SD) for continuous variables as absolute numbers and percentages in parentheses for categorical variables. Chi-square tests were used for the comparison of proportions and Student's t-tests for the comparison of mean values. Pearson correlation analysis was used to determine associations between anthropometric and biochemical variables. Statistical significance was set at $p<0.05$.

\section{Results}

Proportions of children with dyslipidemia and NAFLD were $23.4 \%$ and $31.4 \%$, respectively. The percentage of children with NAFLD was $28.3 \%$ in those without dyslipidemia and $45 \%$ in those with dyslipidemia $(\mathrm{p}=0.015)$. BMI, WC and WHtR were not different between children with and without dyslipidemia (Table 1). WC and WHtR were not different between children with and without NAFLD. However, BMI was significantly greater in those with NAFLD $(\mathrm{p}=0.019)$ (Table 1). A stratified by gender analysis, showed that BMI, WC and WHtR were significantly higher in boys with NAFLD ( $\mathrm{p}=0.009,0.037$ and 0.049 , respectively) (Table 2 ). $\mathrm{WHtR} \geq 0.5$ and HOMA-IR $\geq 3$ was not significantly associated with the presence of dyslipidemia, however HOMA-IR $\geq 3$ was more frequent in children with NAFLD ( $p=0.011$ ) (Table 3 ). A stratified by gender analysis, showed that the aforementioned association was evident only in boys $(\mathrm{p}=0.027)$.

Table I Association between anthropometric measurements and the presence of dyslipidemia and NAFLD ( $\mathrm{P}$ Student's t-test)

\begin{tabular}{lllllllllllll}
\hline \multicolumn{3}{l}{ Dyslipidemia } & \multicolumn{9}{c}{ NAFLD } \\
\hline \multicolumn{3}{l}{ No } & \multicolumn{3}{c}{ Yes } & \multicolumn{3}{c}{ No } & \multicolumn{3}{c}{ Yes } \\
\hline & Mean & SD & Mean & SD & P & Mean & SD & Mean & SD & P \\
\hline BMI $\left(\mathrm{kg} / \mathrm{m}^{2}\right)$ & 26.78 & 3.7 & 26.89 & 3.21 & 0.832 & 26.32 & & 3.44 & 27.4 & 3.81 & 0.019 \\
WC $(\mathrm{cm})$ & 88.91 & 9.6 & 89.49 & 12.1 & 0.747 & 87.79 & & 10.13 & 89.98 & 9.88 & 0.202 \\
WHt Ratio & 0.62 & 0.05 & 0.62 & 0.06 & 0.851 & 0.61 & & 0.05 & 0.62 & 0.05 & 0.182 \\
\hline
\end{tabular}

NAFLD, nonalcoholic fatty liver disease; BMI, body mass index; WC, waist circumference; $\mathrm{WHt}$ Ratio, waist-to-height ratio

Table 2 Association between anthropometric and blood pressure measurements and the presence of NAFLD in boys and girls ( $\mathrm{p}$ Student's t-test)

\begin{tabular}{|c|c|c|c|c|c|c|c|c|c|c|}
\hline & \multicolumn{5}{|c|}{ NAFLD } & \multicolumn{5}{|c|}{ NAFLD } \\
\hline & \multicolumn{5}{|c|}{ Sex: Boys } & \multicolumn{5}{|c|}{ Sex: Girls } \\
\hline & No & & Yes & & & No & & Yes & & \\
\hline & Mean & SD & Mean & SD & $\mathbf{p}$ & Mean & SD & Mean & SD & $\mathbf{p}$ \\
\hline BMI $\left(\mathrm{kg} / \mathrm{m}^{2}\right)$ & 26.68 & 3.31 & 28.54 & 4.02 & 0.009 & 26 & 3.53 & 26.68 & 3.53 & 0.253 \\
\hline WC & 89.66 & 9.08 & 94.4 & 9.08 & 0.037 & 86.21 & 10.77 & 85.73 & 8.83 & 0.844 \\
\hline WHt Ratio & 0.62 & 0.05 & 0.64 & 0.05 & 0.049 & 0.61 & 0.05 & 0.61 & 0.05 & 0.968 \\
\hline
\end{tabular}

NAFLD, nonalcoholic fatty liver disease; BMI, body mass index;WC, waist circumference;WHt Ratio, waist-to-height ratio 
Table 3 Association between anthropometric measurements and HOMA-IR with the presence of dyslipidemia and NAFLD (chi-square test)

\begin{tabular}{|c|c|c|c|c|c|c|c|}
\hline & & \multicolumn{6}{|c|}{ Dyslipidemia } \\
\hline & & \multicolumn{3}{|l|}{ NO } & \multicolumn{3}{|c|}{ Yes } \\
\hline & & $\mathbf{N}$ & $\%$ & & $\mathbf{N}$ & $\%$ & $\mathbf{P} *$ \\
\hline \multirow[t]{2}{*}{ WHt Ratio } & $<0.5$ & 2 & 1.6 & & I & 2.3 & $>0.999$ \\
\hline & $\geq 0.5$ & 127 & 98.4 & & 43 & 97.7 & \\
\hline \multirow[t]{5}{*}{ HOMA-IR $\geq 3$} & $<3$ & 101 & 63.1 & & 28 & 58.3 & 0.549 \\
\hline & $\geq 3$ & 59 & 36.9 & & 20 & 41.7 & \\
\hline & & \multicolumn{6}{|c|}{ NAFLD } \\
\hline & & \multicolumn{2}{|l|}{ NO } & \multicolumn{4}{|l|}{ Yes } \\
\hline & & $\mathbf{N}$ & $\%$ & $\mathbf{N}$ & & $\%$ & $\mathbf{P} *$ \\
\hline \multirow[t]{2}{*}{ WHt Ratio } & $<0.5$ & 2 & 1.9 & I & & 2 & 0.969 \\
\hline & $\geq 0.5$ & 105 & 98.1 & 50 & & 98 & \\
\hline \multirow[t]{2}{*}{ HOMA-IR $\geq 3$} & $<3$ & 91 & 69.5 & 33 & & 50.8 & 0.011 \\
\hline & $\geq 3$ & 40 & 30.5 & 32 & & 49.2 & \\
\hline
\end{tabular}

WHt Ratio, waist-to-height ratio; HOMA-IR, homeostasis model assessment of insulin resistance; NAFLD, nonalcoholic fatty liver disease

\section{Discussion}

In the present cohort, $31.4 \%$ of the participants had elevated ALT and were suspected to have NAFLD. The risk for NAFLD was correlated with dyslipidemia, male gender and with the severity of obesity as evidenced mainly by BMI and insulin resistance. The prevalence of NAFLD varies widely among pediatric populations, ranging from $9 \%$ to $37 \%$ among children and adolescents in different studies, depending on different definitions. ${ }^{2,11}$ This variability is due to the use of different methods of detection, diagnostic criteria, and population selection. ${ }^{11}$ The present findings are consistent with the results of a recent systematic meta-analysis and review by Anderson et al who reported a mean prevalence of NAFLD of $34.2 \%$ in studies based on child obesity clinics. ${ }^{11}$ In the current report, BMI, WC and WHtR were significantly higher in boys with NAFLD $(\mathrm{p}=0.009$, 0.037 and 0.049 , respectively). No significant differences were found in girls. NAFLD seems to be more frequent in boys than in girls. ${ }^{2}$ In the majority of the reported studies, boys outnumber girls in the prevalence of NAFLD and its associated complications. ${ }^{11,12}$ A possible explanation is that sex hormones affect the distribution of fat and muscle with males being more likely to deposit body fat in the intraabdominal area. ${ }^{12}$ NAFLD is reported in all races and ethnicities but certain groups are at increased risk such as Hispanics, Asians, and Africans. ${ }^{11,12}$

The available data suggest that all the factors of the metabolic syndrome are strongly related to NAFLD: hypertension, dyslipidemia, visceral adiposity, insulin resistance and impaired glucose tolerance. ${ }^{2,13}$ It is known that the presence of NAFLD increases with increasing BMI. ${ }^{14}$ Felix et al studied 55 obese children and found a high prevalence of NAFLD. Especially, male gender, increased consumption of refined carbohydrates in the diet, and sedentary lifestyle were significant risk factors for the occurrence of NAFLD ${ }^{15}$ Papandreou et al studied 82 children with obesity and found significantly higher rates of BMI and WC among children with NAFLD. ${ }^{16}$ In this study we demonstrated that the prevalence of HOMA-IR $\geq 3$ was significantly associated with NAFLD $(\mathrm{p}=0.011)$. Dyslipidemia was not significantly correlated with HOMA-IR $\geq 3$ ( $p=0.549$ respectively). However, NAFLD was significantly more prevalent in subjects with dyslipidemia (45\%) than in those without dyslipidemia $(28.3 \%)(p=0.015)$. As shown by a systematic review by D'Adamo et al, NAFLD is associated with insulin resistance and dyslipidemia. ${ }^{17}$ IR is a critical link between MetS and NAFLD in obese children and adolescents. ${ }^{17}$ Sometimes it is even correlated with more severe stages of NAFLD but the exact pathogenic mechanisms are not clearly understood. ${ }^{13,17} \mathrm{Fu}$ et al., ${ }^{18}$ studied 861 children with obesity and reported a positive correlation between NAFLD and components of MetS: hypertension, dyslipidemia, and impaired fasting glucose. Since most pediatric patients with dyslipidemia and NAFLD are obese, the adoption of a physically active lifestyle and gradual weight loss are recommended for the prevention and treatment of these conditions. ${ }^{2,6}$ Early detection and intervention may not only prevent the progress of liver disease, but also reduce the occurrence of metabolic disorders.

The limitations of this study include the bias present in the selection of a sample from a tertiary referral center as well as the absence of liver ultrasound assessment of the severity of NAFLD and the lack of a control group. On the other hand, this study was conducted in Greek children population. Given that prevalence of NAFLD varies widely among children of different race and ethnicity, studies on different ethnic populations contribute to set the appropriate cut off values for more accurate detection of children with fatty liver prospectively.

\section{Conclusion}

The severity of obesity as evidenced mainly by BMI and insulin resistance is clinical indicator of increased risk of dyslipidemia and NAFLD among obese children and adolescents. Moreover, male gender is a risk factor for the development of NAFLD. As most chronic liver diseases are asymptomatic, such data may prove useful in developing models for screening. If NAFLD persists it may lead to irreversible liver damage. Thus children at increased risk should be followed closely and have a more intense program of healthy diet and physical activity, in order to reverse the process. Furthermore, future studies should focus on gender and race differences to ameliorate the understanding of the influence of race and gender on NAFLD risk. 


\section{Acknowledgments}

We want to thank all the nurses and the laboratory staff for their contribution in the care of the patients.

\section{Conflicts of interest}

The authors declare that the research was conducted in the absence of any interests mentioned.

\section{Funding}

None.

\section{References}

1. Styne DM, Arslanian SA, Connor EL, et al. Pediatric obesity—assessment, treatment, and prevention: an Endocrine Society Clinical Practice guideline. JCEM. 2017;102:709-757.

2. Draijer L, Benninga M, Koot B. Pediatric NAFLD: an overview and recent developments in diagnostics and treatment. Expert Rev Gastroenterol Hepatol. 2019;3:447-461.

3. Wieckowska A, Feldstein AE. Nonalcoholic fatty liver disease in the pediatric population: a review. Curr Opin Pediatr. 2005;17:636-641.

4. Marion A, Baker A, Dhawan A.Fatty liver disease in children. Arch Dis Child. 2004;89:648-652.

5. Nobili V, Reale A, Alisi A, et al. Elevated serum ALT in children presenting to the emergency unit: Relationship with NAFLD. Dig Liver Dis. 2009;4:749-752.

6. Vos MB, Abrams SH, Barlow SE, et al. NASPGHAN Clinical Practice Guideline for the Diagnosis and Treatment of Nonalcoholic Fatty Liver Disease in Children: Recommendations from the Expert Committee on NAFLD (ECON) and the North American Society of Pediatric Gastroenterology, Hepatology and Nutrition (NASPGHAN). J Pediatr Gastroenterol Nutr. 2017;64:319-334.

7. Fernandez JR, Redden DT, Pietrobelli A, et al. Waist circumference percentiles in nationally representative samples of African-American, European-American, and Mexican-American children and adolescents. $J$ Pediatr. 2004;145(4):439-444.
8. Tresaco B, Bueno G, Pineda I, et al. Homeostatic model assessment (HOMA) index cut-off values to identify the metabolic syndrome in children. J Physiol Biochem. 2005;61(2):381-388.

9. Kostovski M, Simeonovski V, Mironska K, et al. Metabolic Profiles in Obese Children and Adolescents with Insulin Resistance. Open Access Maced J Med Sci. 2018;6:511-518.

10. Schwimmer JB, Dunn W, Norman GJ, et al. 2010. SAFETY study: alanine aminotransferase cutoff values are set too high for reliable detection of pediatric chronic liver disease. Gastroenterology. 2010;138(4):1357-64 1364.e1-2.

11. Anderson EL, Howe LD, Jones HE, et al. The Prevalence of NonAlcoholic Fatty Liver Disease in Children and Adolescents: A Systematic Review and Meta-Analysis. PLoS One. 2015;10(10):e0140908.

12. Schwimmer JB, McGreal N, Deutsch R, et al. Influence of gender, race, and ethnicity on suspected fatty liver in obese adolescents. Pediatrics. 2005;115(5):e561-e565.

13. Patton HM, Yates K, Unalp-Arida A, et al. Association between metabolic syndrome and liver histology among children with nonalcoholic fatty liver disease. Am J Gastroenterol. 2010;105:2093.

14. Elizabeth LY, Golshan S, Harlow KE, et al. Prevalence of nonalcoholic fatty liver disease in children with obesity. J Pediatr. 2019;207:64-70.

15. Felix DR, Costenaro F, Gottschall CB, et al. Non-alcoholic fatty liver disease (Nafld) in obese children- effect of refined carbohydrates in diet. BMC Pediatr. 201;16(1):187.

16. Papandreou D, Karabouta Z, Pantoleon A, et al. Investigation of anthropometric, biochemical and dietary parameters of obese children with and without non-alcoholic fatty liver disease. Appetite. 2012;59:939944.

17. D'Adamo E, Castorani V, Nobili V. The liver in children with metabolic syndrome. Front Endocrinol. 2019;10:514.

18. Fu JF, Shi HB, Liu LR, et al. Non-alcoholic fatty liver disease: An early mediator predicting metabolic syndrome in obese children? World $J$ Gastroenterol. 2011;17:735-742. 